\title{
Notes on Afrotropical Pyrgodesmidae, 2 (Diplopoda: Polydesmida)
}

\section{Заметки по афротропическим Pyrgodesmidae, 2 (Diplopoda: Polydesmida)}

\author{
S.I. Golovatch ${ }^{1}$, A.R. Nzoko Fiemapong ${ }^{2}$, D. VandenSpiegel ${ }^{3}$ \\ С.И. Головач ${ }^{1}$, А.Р. Нзоко Фьемапонг ${ }^{2}$, А. ВанденШпигель ${ }^{3}$
}

\author{
${ }^{1}$ Institute for Problems of Ecology and Evolution, Russian Academy of Sciences, Leninsky prospekt 33, Moscow 119071 Russia. \\ ${ }^{1}$ Институт проблем экологии и эволюции РАН, Ленинский пр-т, 33, Москва 119071 Россия. \\ ${ }^{2}$ Laboratoire de Zoologie, Université Yaoundé 1, BP812, Cameroun. \\ 2 Лаборатория зоологии, Университет Яунде 1, ВР812 Камерун. \\ ${ }^{3}$ Musée Royal de l'Afrique Centrale, B-3480 Tervuren, Belgique. \\ ${ }^{3}$ Королевский музей Цеитральной Африки, Тервурен, Бельгия.
}

KEY WORDS: millipede, Pyrgodesmidae, taxonomy, new species, new combination, key, Cameroon. КЛЮЧЕВЫЕ СЛОВА: диплопода, Pyrgodesmidae, таксономия, новый вид, новая комбинация, ключ, Камерун.

ABSTRACT. The genera Urodesmus and Monachodesmus are reviewed and shown to comprise four and 18 species, respectively, all keyed, including $U$. cornutus sp.n., M. longicaudatus sp.n. and M. armorum sp.n., all three from Cameroon. In addition, the West African genus Udodesmus is recorded in Cameroon for the first time, being represented there by $U$. camerunensis sp.n., which is unique among congeners in showing $5+5$ undifferentiated lobulations at the anterior margin of the collum.

РЕЗЮМЕ. Даны обзоры родов Urodesmus и Monachodesmus, которые содержат теперь соответственно четыре и 18 видов. Для них всех составлены кдючи, в т.ч. $U$. cornutus sp.n., M. longicaudatus sp.n. и M. armorum sp.n. из Камеруна. Кроме того, западноафриканский род Udodesmus впервые приводится для фауны Камеруна, где он представлен U. camerunensis sp.n., который уникален среди видов рода тем, что у него $5+5$ недифференцированных долей на переднем краю коллума.

\section{Introduction}

The mainly tropical millipede family Pyrgodesmidae is among the largest, but taxonomically perhaps most confused in the entire class Diplopoda, currently counting almost 400 species or subspecies in $170+$ genera, nearly 120 of which are monotypic [Jorgensen, Sierwald, 1910; updated].

The present paper puts on record four new species of Pyrgodesmidae from Cameroon. In this connection, two genera are reviewed and rediagnosed.

\section{Material and methods}

Most of the material treated here derives from the collection of the Musée Royal de l'Afrique Centrale (MRAC), Tervuren, Belgium, with only a few duplicates retained for the collection of the University of Yaoundé 1, Cameroon (UYC) or donated to the Zoological Museum, State University of Moscow (ZMUM), Russia. The samples are stored in $70 \%$ ethanol. Specimens for scanning electron microscopy (SEM) were air-dried, mounted on aluminium stubs, coated with gold and studied using a JEOL JSM-6480LV scanning electron microscope. Photographs were taken with a Leica digital camera Leica DFC 500 mounted on a Leica MZ16A stereomicroscope. Images were processed with the Leica Application Suite software.

\section{Taxonomic part}

On the statuses of Urodesmus Porat, 1894 and Monachodesmus Silvestri, 1927

Urodesmus was one of the earliest genera of Pyrgodesmidae to have been proposed. Porat [1894] compared it directly with Pyrgodesmus Pocock, 1892, and described two species therein: Urodesmus erinaceus Porat, 1894 and U. sexcarinatus Porat, 1894, both from Cameroon. However, Urodesmus was properly typified only by Silvestri [1896] who not only beautifully redescribed U. erinaceus from type material [Silvestri, 1927], but also designated it as the type species. Verhoeff [1936] ignored the above typification, as well as a couple of varieties which Silvestri [1927] had distinguished in U. erinaceus: U. e. var. nigeriensis, from Nigeria, and $U$. e. var. minor, from Fernando Po Island. 
From the very start, $U$. erinaceus was readily distinguished from $U$. sexcarinatus by the presence of a paramedian pair of very high, sometimes vaguely bilobed, basally subcontiguous, anteriorly directed, roundish tubercles located in the central part of the collum behind a nearly straight anterior margin with $5+5$ rather superficial lobulations [Porat, 1894; Silvestri, 1927]. Each following metatergite in $U$. erinaceus shows a paramedian pair of similarly high, often heavily earthencrusted, apically largely vaguely bi- or trilobate, mostly subvertical and clearly separated tubercles growing increasingly strongly inclined caudad only in the last few segments. The paraterga are prominent, set very low, subhorizontal, lying almost level to venter, vaguely bi- or trilobate at the lateral margin, devoid of antero- and caudolateral lobulations. The pore formula is normal $(5,7,9,10,12,13,15-19)$, very evident porosteles are only present in paraterga 5, 7, 9, 10, 12, 13, 15 and 16. The dorsal protuberances of segment 19 are clearly directed caudad, short to moderately elongate, even if clearly split medially, then leaving the tip of the epiproct fully or nearly exposed in dorsal view. The gonopods in $U$. erinaceus are deeply sunken inside a prominent gonocoel, the telopodites are massive, complex, deeply bi- or tripartite, including a solenomere.

Such a concept of Urodesmus has since remained largely intact. Only Attems [1940] correctly added Stylodesmus camerunensis Silvestri, 1927, from Cameroon, while Stylodesmus horridus Cook, 1895, and its further two congeners from western Africa, have been removed [Mauriès, Maurin, 1981; Golovatch, VandenSpiegel, 2014]. As a result, Urodesmus currently encompasses only the following few species: Urodesmus erinaceus Porat, 1894 (the type species), from Cameroon, Fernando Po Island, and Nigeria, U. camerunensis (Silvestri, 1927), from Cameroon, and U. simplex Silvestri, 1927, from Nigeria.

Urodesmus serratus Verhoeff, 1936, described from Sri Lanka as the type, and sole constituent, species of the subgenus Attemsocyphus Verhoeff, 1936 [Verhoeff, 1936; Attems, 1940], seems best to be removed from Urodesmus, albeit largely based on zoogeographical grounds. Here we follow Verhoeff [1937] not only because he promoted Attemsocyphus to the rank of a full genus, but he also provided meaningful illustrations of the collum, caudal body end and gonopod structure to further document the differences of $A$. serratus from Urodesmus spp. These diffferences also concern the presence of intercalary granulations between the main crests which never occur in the African counterparts.

One new species is added here to Urodesmus.

In contrast, the collum in $U$. sexcarinatus shows $2+2$ and $2+2$ central, rather small tubercles behind an indistinctly $5+5$ lobulate anterior margin [Porat, 1894]. In addition, metaterga 2-4 each has two, following ones three, transverse rows of small round tubercles, the dorsal crests being clearly higher than the lateral crests which lie above the paraterga. What seems espe- cially remarkable, however, is the presence of caudad rather strongly elongated, coniform crests 19 which conceal the tip of the epiproct from above.

Such conditions readily remind of those observed in the rather large, mostly Afrotropical genus Monachodesmus Silvestri, 1927. The main differences from Urodesmus lie in the ornamentation of the collum and following metaterga, these in the latter genus showing $1+1$ high dorsal protuberances which are rather weakly split on segment 19 and usually fail to conceal the tip of the epiproct from above (Figs 1A, 2A-I). The distribution patterns of ozopores and tergal ornamentations along the body remain basically the same, only porosteles in Monachodesmus are exceptionally absent from segment 16. Each dorsal crest on segments 2-4 is composed of two tubercles, thereafter, like all lateral crests, of three tubercles. The paraterga in Monachodesmus species are only seldom poorly developed, nearly wanting, while many species show small intercalary granulations between and below the main $(2+2)$ metatergal crests. In addition, the epiproct in Monachodesmus is often fully concealed from above by the medially fused ultimate dorsal crests, although in some species it remains up to fully exposed due to the rather short and independent ultimate crests. All possible transitions in the degree of fusion and caudad protrusion of the ultimate crests are thereby observed.

In general, the ultimate crests (usually $19^{\text {th }}$, depending on the number of body segments) being very considerably elongated/extended caudad is a common trait among tropical Pyrgodesmidae. There can hardly be any doubt that this condition may have appeared many times in the course of the family's evolution. Putting special emphasis on this character alone has resulted in such speciose artificial assemblages as the genera $L o$ phodesmus Pocock, 1892, Myrmecodesmus Silvestri, 1910, Monachodesmus and some others. Thus, Lophodesmus is actually based on L. pusillus Pocock, 1894, a species from Flores, Indonesia, but numerous congeners have since been described not only from tropical parts of Asia, but also from Africa and, especially, America. Hoffman [1999] emphasized that noone must take Lophodesmus seriously at the present. The situation concerning Myrmecodesmus seems to be slightly better, as the genus is restricted to the Americas [Hoffman, 1999], whereas Monachodesmus remains largely Afrotropical, albeit a species from the Philippines has also been included therein [e.g. Attems, 1940].

We suggest treating all Afrotropical species formally described in or assigned to Lophodesmus as actually belonging to Monachodesmus. Among the African Lophodesmus, only L. cristulifera Brolemann, 1920, from Zanzibar, and L. escherichii Silvestri, 1911, from Eritrea, show small intercalary granulations and/or caudal lobulations between the main crests [Silvestri, 1911; Brolemann, 1920]. Both these species are newly transferred to Monachodesmus, comb.n. One of the new species of Monachodesmus described below also has intercalary granulations and caudal lobulations, but nei- 
ther caudo- nor anterolaterals are observed on the paraterga in Monachodesmus species. Urodesmus sexcarinatus Porat, 1894, which has been transferred to Lophodesmus since Attems [1940], likewise seems to be a species of Monachodesmus, comb.n. As regards the status of Stegodesmus leonis Cook, 1896, from Sierra Leone, its description is too concise to properly allocate that species in a genus [Cook, 1896]. Attems [1940] listed it among unidentifiable Lophodesmus spp.

The following species are attributed to Monachodesmus: M. feae Silvestri, 1927, the type species from Principe Island, São Tome \& Principe, M. escherichii (Silvestri, 1911), comb.n. ex Lophodesmus, from Eritrea, M. cristulifera (Brolemann, 1920), comb.n. ex Lophodesmus, from Zanzibar, M. conquisitus (Silvestri, 1927), from Ghana, M. angustus (Silvestri, 1917) and M. tardus (Silvestri, 1917), both from Nigeria, $M$. subangustus (Silvestri, 1927), comb.n. ex Lophodesmus, from Guinea-Bissau, M. lentus Silvestri, 1927. and M. spurcus Silvestri, 1927, both from Cameroon, M. artatus Mauriès et Maurin, 1981, M. abijiensis Mauriès et Maurin, 1981, and M. unus Mauriès et Maurin, 1981, all three from Côte d'Ivoire, M. odiosus Demange et Mauriès, 1975, from Côte d'Ivoire and Guinea, M. silvestrii Mauriès, 1968, from Gaboon, as well as M. lundanus (Chamberlin, 1951), and M. albus Kraus, 1958, both from Angola [Silvestri, 1917, 1927; Chamberli, 1951; Kraus, 1958; Mauriès, 1968; Demange, Mauriès, 1975; Mauriès, Maurin, 1981]. Emphasizing the similarities in gonopod structure alone, Mauriès \& Maurin [1981] accepted the subgenus Monachodesmus s.str. to encompass $M$. feae, M. angustus, M. albus and probably also M. lentus, as opposed to the subgenus Lophoporus Attems, 1931, which those authors revived. Attems [1931] had erected, but quickly abandoned it altogether [Attems, 1940]. According to Mauriès \& Maurin [1981], Lophoporus contains the following species: $M$. tardus (the type species of the subgenus), $M$. spurcus, $M$. conquisitus, $M$. silvestrii, $M$. odiosus, M. artatus, M. abijiensis and M. unus, which all share a long slender branch on the gonopod femorite. Indeed, it seems that only the gonopod conformation rather than peripheral, somatic characters can provide real clues for formally splitting the genus into natural species groups. This is a difficult task though, because the gonopods are mostly complex, homologies of certain parts too often being not so easy to trace.

Below we add another two new congeners.

\section{Species descriptions}

\section{Urodesmus cornutus sp.n.}

Figs 1A, 2, 3.

HOLOTYPE $O^{7}$ (MRAC 22656), Cameroon, Campus Université Yaoundé 1, $\mathrm{N} 03^{\circ} 53^{\prime}$, E $011^{\circ} 30^{\prime}, 860 \mathrm{~m}$ a.s.1., 17.III.2014, leg. A. Nzoko.

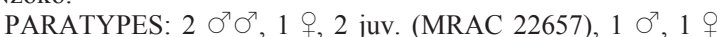
(MARC 22662), $3 \mathrm{O}^{7} \mathrm{O}^{7}, 1$ +, 11 juv. (UYC), $10^{x}, 1$, 1 juv. (ZMUM p2664), same data, together with holotype; $10^{7}, 2$ 우, 1 juv. (UYC), same locality, 14.III.2014; $10^{7}, 2$ 우 (UYC), same locality, 28.IV.2014; $2 \sigma^{7} \sigma^{7}, 3$ $9+, 11$ juv. (MRAC 22660), same locality, 23.IV.2014; 1 (UYC), same locality, 4.VI.2014; same locality, $1 \mathrm{O}^{7}, 6$ 오 (MRAC 22658), same locality, 6.X.2014; 2 오, 25 juv. (MRAC 22659), same locality, 28.V.2015, all leg. A. Nzoko. NON-TYPES: 1 + (MRAC 22665), Cameroon, Zamakoué, N

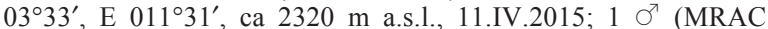
22666), same locality, 28.IV.2014; 2 우 (MRAC 22664), Cameroon, Ongot, $\mathrm{N} 03^{\circ} 51^{\prime}$, E $011^{\circ} 25^{\prime}$, ca 810 m a.s.1., 19.X.2014; 1 우 (MRAC 22666), same locality, 18.IV.2014; 1 \% (MRAC 22663), same locality, 19.X.2014; 1 juv. (UYC), same locality, 7.II.2015; 1 ㅇ (UYC), same locality, 28.IV.2015; 1 juv. fragment (UYC), Cameroon, Egoutadjap, $\mathrm{N} 02^{\circ} 42^{\prime}$, E $011^{\circ} 03^{\prime} 07$, ca $2020 \mathrm{~m}$ a.s.1., 11.IV. 2015; 1 juv. (MRAC 22661), same locality, 19.VII.2014, all leg. A. Nzoko.

NAME. To emphasize the collum bearing two strong, forward directed, paramedian protuberances which clearly overhang the collum's anterior margin and give the animal a highly characteristic, cornuted appearance; adjective.

DIAGNOSIS. Differs mostly by $\sigma^{7}$ prefemur 4 showing a conspicuous distoventral trichostele, the tip of the epiproct being fully concealed from above by paraterga 19, as well as in mesal branch $\mathbf{D}$ of the gonopod telopodite being considerably higher than lateral branch C. See also Key below.

DESCRIPTION. Length of adults ca 11-15, width of midbody pro- and metazonae $1.2-1.7$ and 2.0-3.2, respectively $\left(\mathrm{O}^{7},+\right)$. Holotype ca $13 \mathrm{~mm}$ long, 1.3 and $3.0 \mathrm{~mm}$ wide on pro- and metazonae, respectively. $\sigma^{\top} O^{7}$ usually slightly smaller than 우. General coloration in alcohol light yellow-olive green, usually with heavily earth-encrusted, reddish to brown margins of paraterga, most if not all dorsal protuberances (MD). Cleaner specimens, usually juveniles, from pallid to yellowish. Antennae, venter and legs yellowish.

Head smooth, roundish, genae square; clypeolabral region very finely and densely setose, epicranial suture visible only in occipital region; interantennal isthmus about twice as large as diameter of antennal socket; vertigial region with two elongated fields of fine microgranulations along epicranial suture (Fig. 2D). Antennae medium-sized, clavate, C-shaped, each lying inside a distinct groove, closely appressed to head, with small compact apicodorsal groups of bacilliform sensilla on antennomeres 5 and 6; the former clearly the longest and largest (Fig. 2D).

Body with 20 segments $\left(\mathrm{O}^{7},+\right.$ ) . In width, head $<<$ collum $=$ segment $2<3<4<5=15$; thereafter body gradually tapering towards telson. Body polydesmoid, with very broad, subhorizontal, wing-shaped paraterga set very low (nearly level to venter), usually faintly uptuned laterad (Figs 1A, 2A-I). Collum flabellate, very wide, fully covering the head from above; anterior margin nearly straight, with $5+5$ equal and modest lobulations, central region with $1+1$ very high, fingershaped, often vaguely bifid, rounded, inclined more forward than dorsad, thick protuberances; the latter subcontiguous basally, about as wide as midbody paraterga, both clearly overhanging collum's anterior margin; neither intercalary granulations nor (caudo)lateral lobulations (Figs 1A, 2A, D, H). Parater- 

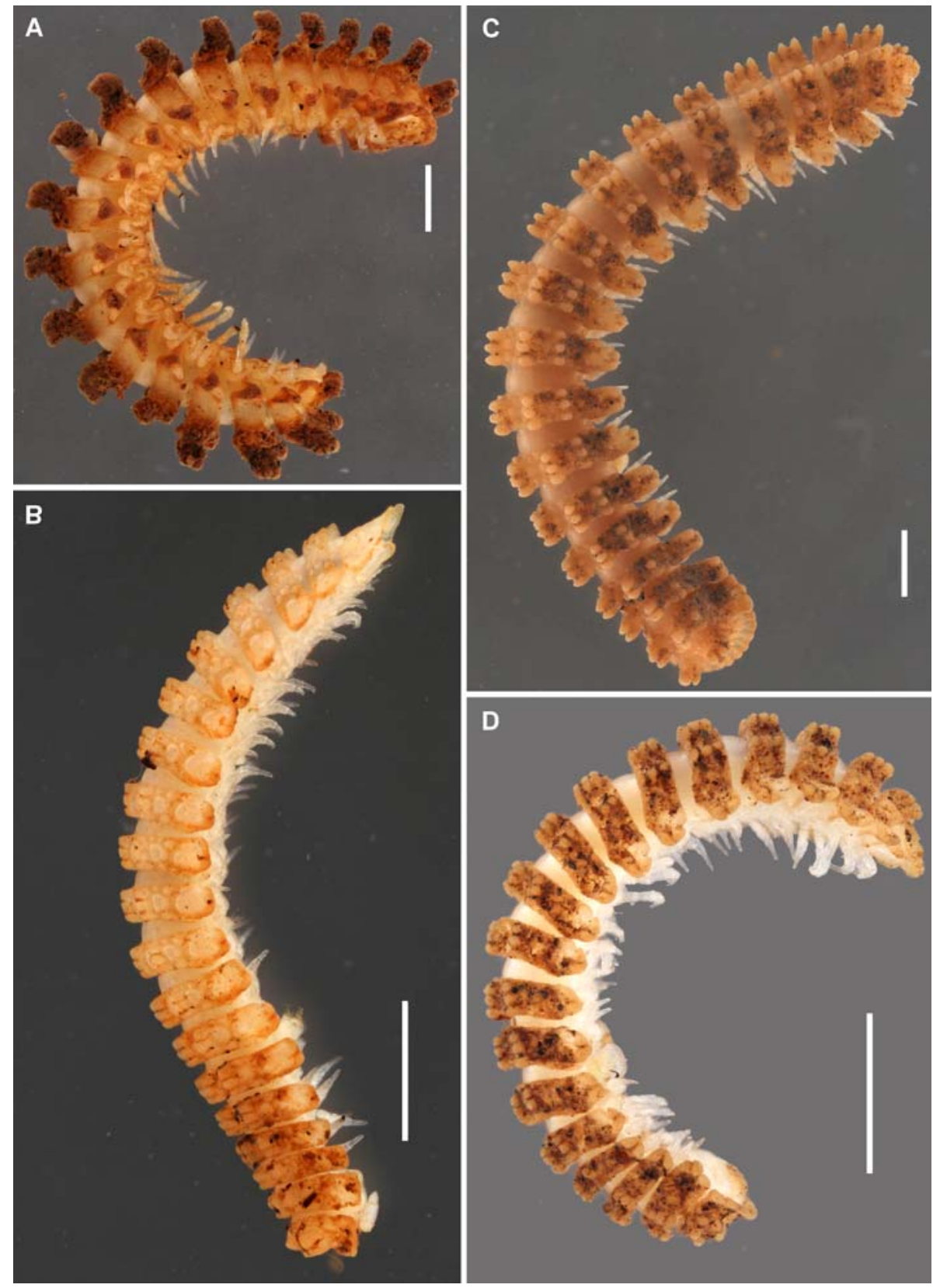

Fig. 1. Habituses of Urodesmus cornutus sp.n. (A), Monachodesmus longicaudatus sp.n. (B), M. armorum sp.n. (C) and Udodesmus camerunensis sp.n. (D), $O^{7}$ paratype, $O^{7}$ paratype, $O^{7}$ holotype and $O^{7}$ holotype, lateral views, respectively. Scale bars: $1.0 \mathrm{~mm}$.

Рис. 1. Внешний вид Urodesmus cornutus sp.n. (А), Monachodesmus longicaudatus sp.n. (В), M. armorum sp.n. (C) и Udodesmus

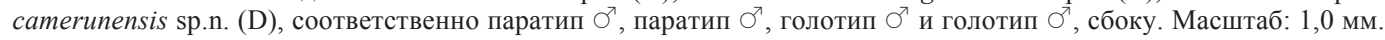

ga 2 slightly enlarged, lateral margin vaguely trilobate, sides strongly declivous to subvertical; dorsal protuberances (MD) on postcollum metaterga similarly high, clearly separated, sigmoid in lateral view, mostly subvertical; starting with paraterga 3 , lateral margin vaguely bilobate, mostly subrectangular at anterior and posterior corners; neither intercalary granulations nor caudolateral, nor anterolateral lobulations, nor tergal setae (Figs 1A, 2A-I). Metaterga strongly convex (Fig. 2G), with a usual cerategument layer on top of microvilli; middle parts sometimes also encrusted with earth ma- terial, with neither bosses nor areations. Pore formula normal: $5,7,9,10,12,13,15-19$; very evident, coniform, simple porosteles present only in paraterga 5,7 , $9,10,12,13,15$ and 16 , in segments 17-19 opening flush on tergal surface, always lying on caudal lobulation near caudal corner (Fig. 2A-J). MD growing increasingly inclined caudad on segments $17-19$, penultimate protuberances overhanging and reaching tips of ultimate ones (Figs 1A, 2C, F, I). Limbus very finely crenulate-denticulate (Fig. 2K). Epiproct completely, but rather little concealed from above between ultimate 

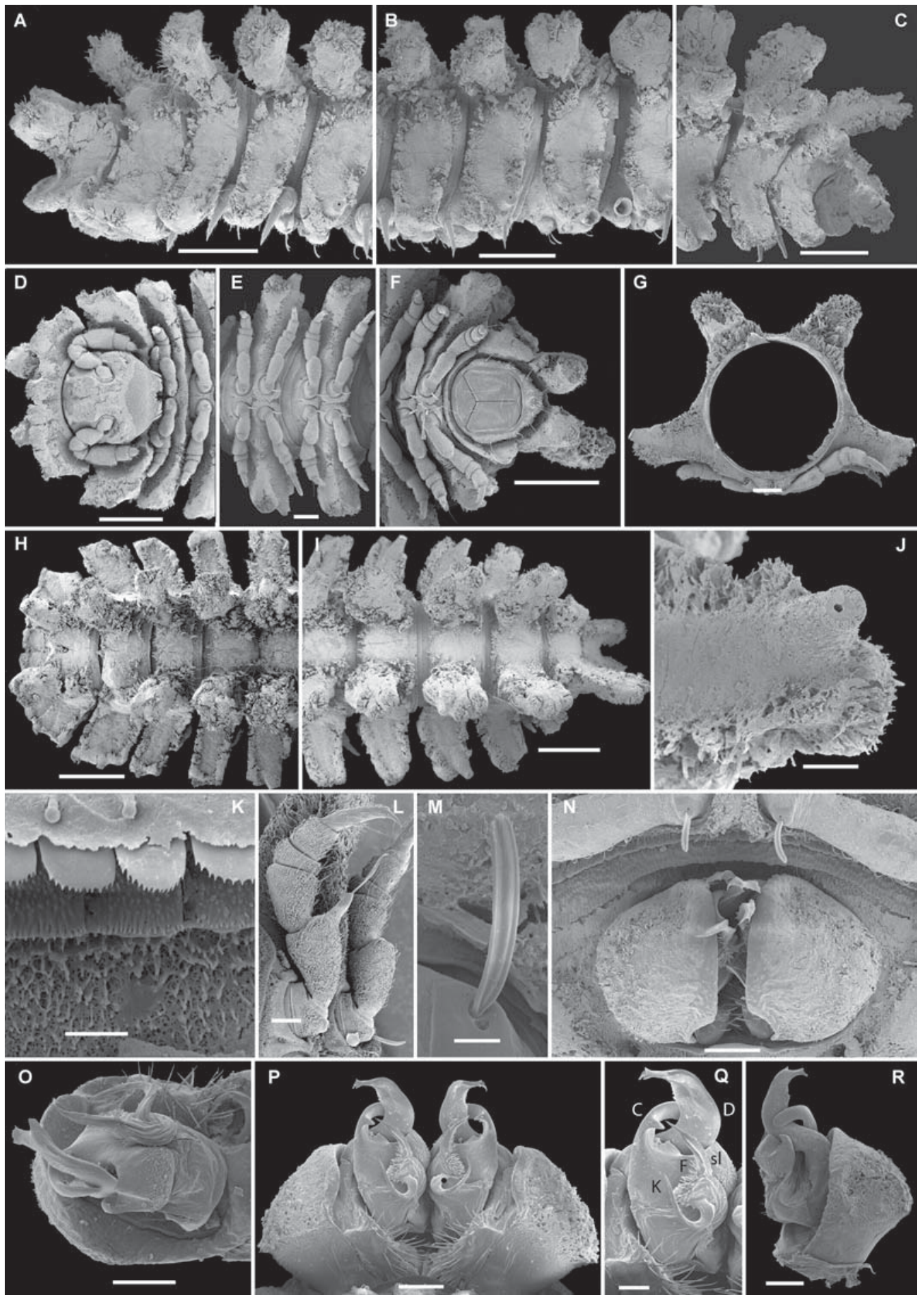

Fig. 2. SEM micrographs of Urodesmus cornutus sp.n., O paratype. A, D \& $\mathrm{H}$ - anterior part of body, lateral, ventral and dorsal views, respectively; B \& E - midbody segments, lateral and ventral views, respectively; C, F \& I - posterior part of body, lateral, ventral and dorsal views, respectively; $\mathrm{G}$ - cross-section of a midbody segment, caudal view; J - poriferrous paratergite with porostele, dorsal view; $\mathrm{K}$ - limbus and adjacent microstructures; $\mathrm{L}$ - legs $4 \& 5$, ventral view; $\mathrm{M}$ - seta on coxa 2, ventral view; $\mathrm{N} \& \mathrm{P}$ - both gonopods in situ, ventral and caudal views, respectively; O - left gonopod, mesal view; Q \& R - right gonopod, caudal and sublateral views, respectively. Scale bars: 0.5 (A-D, F, H, I), $0.2(\mathrm{E}), 0.1(\mathrm{~J}, \mathrm{~L}, \mathrm{~N}), 0.02(\mathrm{M}) \& 0.01 \mathrm{~mm}(\mathrm{~K})$. Designations in text.

Рис. 2. Фотографии SEM Urodesmus cornutus sp.n., паратип О'. А, D, H - передняя часть тела, соответственно сбоку, снизу и сверху; В, Е - среднетуловищные сегменты, соответственно сбоку и снизу; С, F, I - задняя часть тела, соответственно сбоку, снизу и сверху; $\mathrm{G}$ - поперечный срез среднетуловищного сегмента, сзади; J — несущий пору паратергит с поростелем, сверху; K лимбус и смежные микроструктуры; L - ноги 4 и 5 , снизу; M - щетинка на тазике 2, снизу; N, P - оба гонопода на месте, соотваетственно снизу и сзади; O - левый гонопод, изнутри; $\mathrm{Q}, \mathrm{R}$ - правый гонопод, соответственно сзади и почти сбоку. Масштаб: 0,5 (A-D, F, H, I), 0,2 (E), 0,1 (J, L, N), 0,02 (M) и 0,01 мм (K). Обозначения в тексте. 


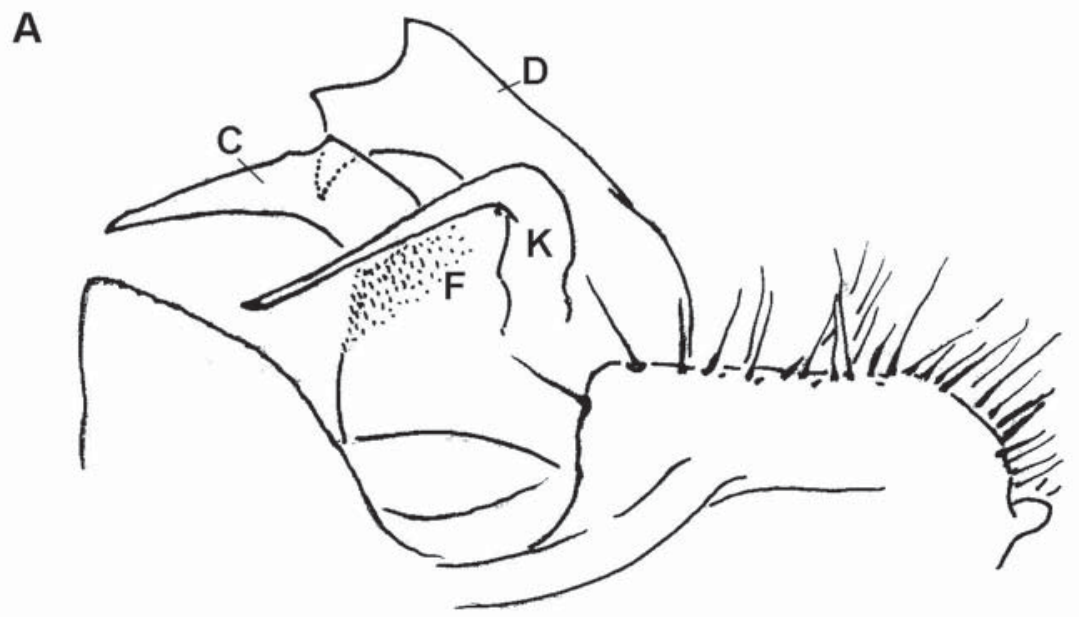

B

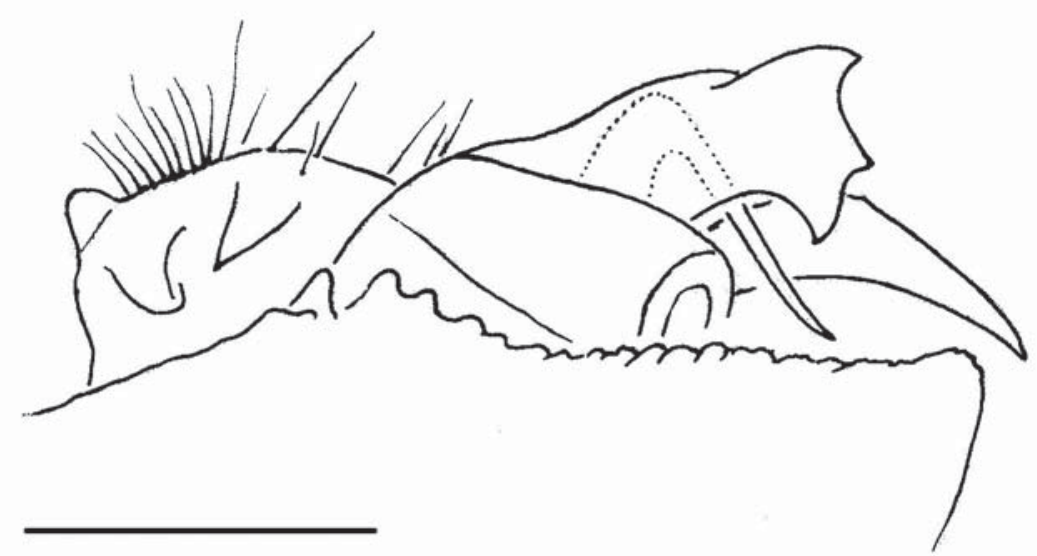

Fig. 3. Left gonopod of Urodesmus cornutus sp.n., ơ paratype. A \& B, mesal and lateral views, respectively. Scale bar: $0.1 \mathrm{~mm}$. Designations in text.

Рис. 3. Левый гонопод Urodesmus cornutus sp.n., паратип О’. А, В, соответственно изнутри и сбоку. Масштаб: 0,1 мм. Обозначения в тексте.

protuberances (Fig. 2C, F, I). Hypoproct subtriangular, with $1+1$ caudal setae devoid of knobs (Fig. 2F).

Sterna narrow, deeply impressed along axis, without modifications, sparsely setose (Fig. 2E, F). Epigynal ridge behind + coxae 2 very low, rounded on sides. Legs relatively short and stout, not extending beyond lateral edges of paraterga, only tarsi particularly slender; $\sigma^{7}$ prefemur 4 with a prominent distoventral trichostele (Fig. 2L); larger setae on $\sigma^{T}$ coxae modified, ribbed (Fig. 2M, N). Gonapophyses on $\sigma^{7}$ coxae 2 vestigial (Fig. 2D).

Gonopod aperture subcordiform, broad, nearly as wide as prozona 7 (Fig. 2N). Gonopods (Figs 2N-R, 3) rather simple; coxae subglobose, large, microgranulate and micropilose laterally, with usual long cannulae and a rather deep gonocoel; each telopodite deeply bipartite, on caudal face with a characteristically curved pseudoflagelloid process $(\mathbf{K})$ at base of a microfimbriate/ micropilose field and a lacinia (F) marking the end part of a solenomere (sl); lateral branch (C) sublaciniate, acuminate, subtending on lateral side only a slightly longer, but more massive, tridentate mesal branch (D).

REMARKS. Using the various gonopod elements as designated by Silvestri [1927] for U. erinaceus, all of its main structures can easily be distinguished: a conspicuous pseudoflagelloid process $(\mathbf{K})$ situated on the caudal face at the base of a solenomere (sl) which is laciniate and fimbriate. Both main branches of the telopodites which only slightly extend beyond the gonocoel are intricately interlocked, the lateral branch $(\mathbf{C})$ subtending the larger mesal branch (D).

The following key can serve to separate all four currently known Urodesmus species:

1(2) 1+1 dorsal protuberances on collum nearly fully fused, dorsal protuberances (MD) on segment 18 far less strongly inclined caudad, leaving those on $19^{\text {th }}$, as well as telson fully exposed in dorsal view.....U. camerunensis 2(1) $1+1$ dorsal protuberances on collum fully separated, these on segment 18 far more strongly inclined caudad, in dorsal view covering much or all of those on $19^{\text {th }}$, sometimes entire telson as well. 

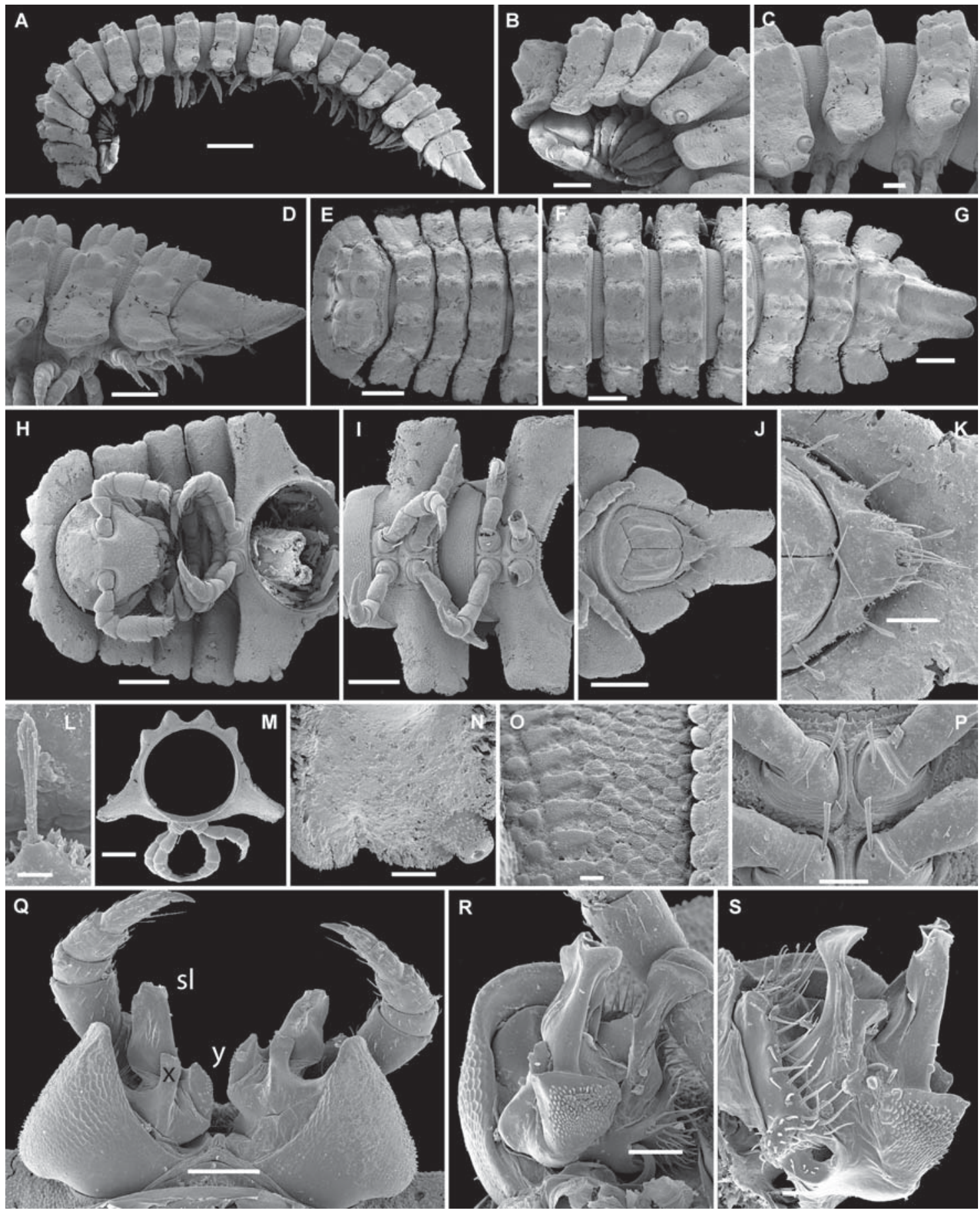

Fig. 4. SEM micrographs of Monachodesmus longicaudatus sp.n., Ơ paratype. A — habitus, lateral view; B, E \& H — anterior part of body, lateral, dorsal and ventral views, respectively; C, F \& I - midbody segments, lateral, dorsal and ventral views, respectively; D, G \& $\mathrm{J}$ - posterior part of body, lateral, dorsal and ventral views, respectively; K — epiproct, ventral view; L — distoventral coxal seta, ventral view; $\mathrm{M}$ - cross-section of a midbody segment, caudal view; $\mathrm{N}$ - poriferrous paratergite with porostele, dorsal view; $\mathrm{O}$ - limbus and adjacent microstructures; P - midbody sterna and coxae with setae, ventral view; Q — both gonopods in situ and legs 7, caudal view; R right gonopod, ventrocaudal view; S - left gonopod, mesal view. Scale bars: 0.5 (A), 0.2 (B, D-J, M), 0.1 (C, O-S), 0.05 (K, N), 0.02 (M), \& $0.01 \mathrm{~mm}(\mathrm{~L})$. Designations in text.

Рис. 4. Фотографии SEM Monachodesmus longicaudatus sp.n., паратип О’. А - общий вид, сбоку; В, Е, Н — передняя часть тела, соответственно сбоку, сверху и снизу; С, F, I — среднетуловищные сегменты, соответственно сбоку, сверху и снизу; D, G, J задняя часть тела, соответственно сбоку, сверху и снизу; $\mathrm{K}$ - эпипрокт, снизу; L — дистовентральная щетинка на тазике, снизу; M - поперечный срез среднетуловищного сегмента, сзади; $\mathrm{N}$ - несущий пору паратергит с поростелем, сверху; O — лимбус и смежные микроструктуры; P - среднетуловищные стерниты и тазики со щетинками, Q - оба гонопода на месте и ноги 7, сзади; $\mathrm{R}$ - правый гонопод, одновременно снизу и сзади; S - левый гонопод, изнутри. Масштаб: 0,5 (A), 0,2 (B, D-J, M), 0,1 (C, O-S), $0,05(\mathrm{~K}, \mathrm{~N}), 0,02(\mathrm{M})$ и 0,01 мм (L). Обозначения в тексте. 
3(4) Tips of $1+1$ dorsal protuberances on collum single, not subdivided even vaguely. MD on segment 19 especially strongly inclined caudad, clearly overreaching tips of paraterga 19........................................... U. simplex

4(3) Tips of $1+1$ dorsal protuberances on collum, often however vaguely, subdivided into two or four. MD on segment 19 at most reaching tips of paraterga 19...... 5

5(6) $\sigma^{7}$ prefemur 4 with a conspicuous distoventral trichostele (Fig. 2L). Tip of epiproct fully concealed from above by paraterga 19 (Fig. 2F, I). Mesal branch $\mathbf{D}$ of gonopod telopodite considerably higher than lateral branch $\mathbf{C}$ (Figs 2P-R, 3)........................... Urodesmus cornutus sp.n.

6(5) $O^{7}$ prefemur 4 unmodified. Tip of epiproct fully exposed from above between paraterga 19. Mesal branch D of gonopod telopodite usually about as high as lateral branch $\mathbf{C}$. If branch $\mathbf{D}$ clearly longer than $\mathbf{C}$, then dorsal protuberances and paraterga considerably smaller, each only about as broad as high (U. e. var. minor).

Urodesmus erinaceus

\section{Monachodesmus longicaudatus sp.n.}

Figs 1B, 4, 5.

HOLOTYPE O (MRAC 22667), Cameroon, Campus Université Yaoundé 1, $\mathrm{N} 03^{\circ} 53^{\prime}$, E $011^{\circ} 30^{\prime}, 860 \mathrm{~m}$ a.s.1., 8.III.2014, leg. A. Nzoko.

PARATYPES: $10^{7}, 1$ (MRAC 22670), same locality, 7.VII. 2014; $1 \sigma^{7}$ fragment, 19 (MRAC 22673), $2 \sigma^{\top} \sigma^{7}, 1+1$ frament (UYC), same locality, 28.V.2014; $1 \mathrm{O}^{7}, 1$ ㅇ (ZMUM $\rho 2666$ ), same locality, 24.IV.2014; 1 \% subadult (MRAC 22672), same locality, 12.IV.2014; 2 ㅇ, 6 juv. (MRAC 22671), same locality, 6.X.2014; 1 fragment (without caudal part of body) (MRAC 22668), same locality, 28.V.2014, all leg. A. Nzoko.

NON-TYPES: 1 fragment (posterior part of body) (UYC), same locality, 17.III.2014; 1 +, 2 subadults (MRAC 22669), Cameroon, Ongot, $\mathrm{N} 03^{\circ} 51^{\prime}$, E $011^{\circ} 25^{\prime}$, ca 810 m a.s.1., 19.VII.2014, all leg. A. Nzoko

NAME. To emphasize the unusually long and caudally extended paraterga 19; adjective.

DIAGNOSIS. Differs mostly in the tip of the epiproct being fully concealed from above by the extremely long and medially fused dorsal crests (MD) on segment 19 , coupled with the gonopod telopodite being deeply bipartite, divided into a shorter, caudal, simple, spade-shaped process ( $\mathbf{x})$ and a higher, similarly simple, plate-like, anterolateral solenomere (sl). See also Key below.

DESCRIPTION. Length of adults ca $6-8$, width of midbody pro- and metazonae $0.6-0.8$ and $1.2-1.3 \mathrm{~mm}$, respectively $\left(\sigma^{7}, q\right)$. Holotype ca $6 \mathrm{~mm}$ long, 0.7 and $1.2 \mathrm{~mm}$ wide on pro- and metazonae, respectively. $\sigma^{\top} \sigma^{7}$ usually slightly smaller than +9 . General coloration in alcohol light beige, usually with earth-encrusted, reddish to brown margins of paraterga and sometimes also dorsal crests (Fig. 1B). Cleaner specimens, usually juveniles, from pallid to yellowish. Vertigial paramedian regions above antennae usually yellowish.

Head smooth, roundish, genae square; clypeolabral region very finely and densely setose, epicranial suture visible only in occipital region; interantennal isthmus about twice as large as diameter of antennal socket; vertigial region with two elongated fields of fine microgranulations along epicranial suture (Fig. 4H). Antennae medium-sized, clavate, C-shaped, each lying

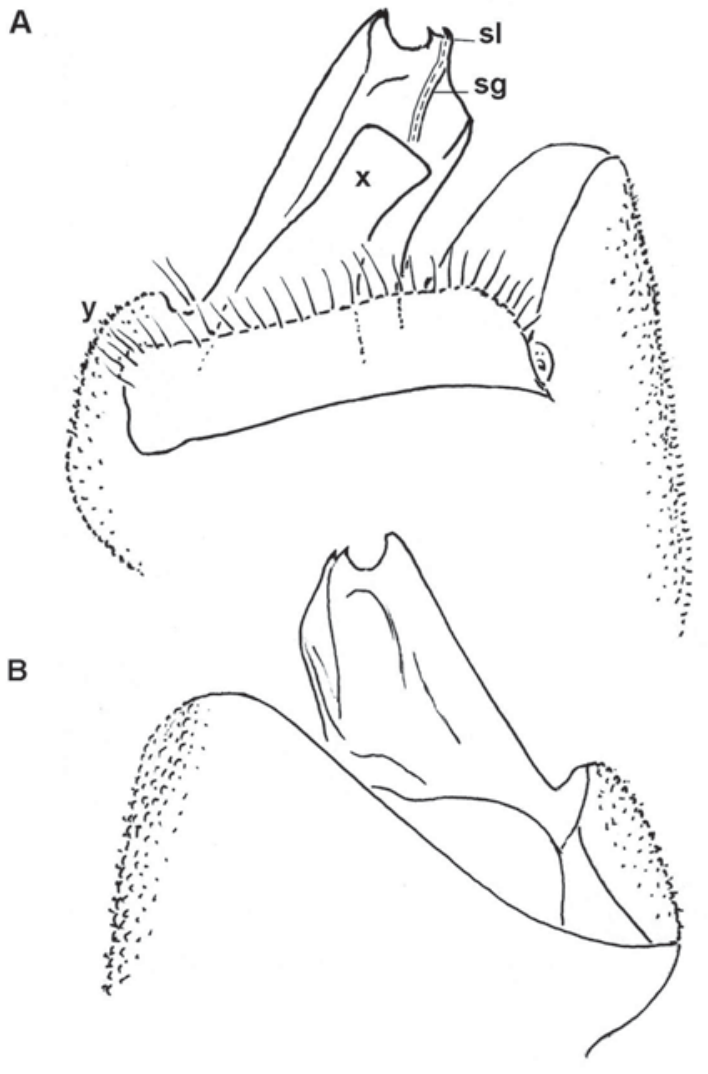

Fig. 5. Left gonopod of Monachodesmus longicaudatus sp.n., $\sigma^{7}$ paratype. A \& B, submesal and sublateral views, respectively. Scale bar: $0.1 \mathrm{~mm}$. Designations in text.

Рис. 5. Левый гонопод Monachodesmus longicaudatus sp.n., паратип $\sigma^{\top}$. А, В, соответственно почти изнутри и почти сбоку. Масштаб: 0,1 мм. Обозначения в тексте.

inside a distinct groove, closely appressed to head, with small compact apicodorsal groups of bacilliform sensilla on antennomeres 5 and 6; the former clearly the longest and largest (Fig. $2 \mathrm{H}$ ).

Body with 20 segments $\left(O^{\top},+\right.$ ) $)$. In width, head $<<$ collum $=$ segment $2<3<4<5=15$; thereafter body gradually tapering towards telson. Body polydesmoid, with broad, moderately declivous, wing-shaped paraterga set very low (nearly level to venter) (Figs 1B, 4A$\mathrm{G}, \mathrm{M}, \mathrm{N}$ ). Collum flabellate, very wide, fully covering the head from above; anterior margin nearly straight, with $5+5$ equal and faint lobulations, only $2+2$ lateralmost lobulations located at a clearly rounded margin; central region with $2+2$ high, distinctly bifid, basally subcontiguous crests, these being inclined more forward than dorsad, not overhanging collum's anterior margin; neither intercalary granulations nor (caudo)lateral lobulations (Figs 1B, 4A, B, H). Lateral margin of paraterga 2 trilobate, following ones bilobate; sides strongly declivous to subvertical; MD on postcollum metaterga similarly high, narrow, clearly separated, mostly subvertical, MD only slightly highter than lateral crests (DL), largely rather vaguely trilo- 
bate; starting with paraterga 2, lateral margin mostly subrectangular at anterior and posterior corners; neither intercalary granulations nor caudolateral, nor anterolateral lobulations, nor tergal setae (Figs 1B, 4A-I, M, N). Metaterga strongly convex (Fig. 4M), with a usual cerategument layer on top of microvilli (Fig. 4K, N); middle parts sometimes also encrusted with earth material, with neither bosses nor areations. Pore formula normal: $5,7,9,10,12,13,15-19$; very evident, coniform, simple porosteles present only in paraterga $5,6,8,9,11,12$, $14,15 \& 16$, in segments $17-19$ opening flush on tergal surface, always lying on caudal lobulation near caudal corner (Fig. 4A-G, N). MD growing only slightly inclined caudad on segments 17 and 18, on segment 19 suddenly and greatly extended backwards, fused before tip into a deeply bilobate end which is nearly as long as telson (Figs 1B, 4C, G, I). Limbus very finely crenulate (Fig. 4O). Epiproct completely and very strongly concealed from above between medially fused ultimate crests (Fig. 4C, D, I, K). Hypoproct subtriangular, with 1+1 caudal setae devoid of knobs (Fig. 4I).

Sterna narrow, deeply impressed along axis, without modifications, sparsely setose (Fig. 4H, I, M, P). Epigynal ridge behind $q$ coxae 2 very low, rounded on sides. Legs relatively short and stout, not extending beyond lateral edges of paraterga, only tarsi particularly slender; larger setae on $\sigma^{7}$ coxae modified, ribbed (Fig. 4L, P). Gonapophyses on $\sigma^{7}$ coxae 2 vestigial.

Gonopod aperture subcordiform, broad, nearly as wide as prozona 7. Gonopods (Figs 4Q-S, 5) rather simple; coxae subglobose, large, microgranulate and micropilose laterally, with usual long cannulae and a rather deep gonocoel; each telopodite deeply bipartite, on caudal face with a shorter, characteristically spadeshaped process (x) lying opposite a smaller, rounded, mesal, laterally micropapillate bulge (y) and a far larger, but simple, anterolateral, apically faintly microdenticulate, plate-like solenomere (sl) supporting a seminal groove (sg) on its caudal face.

\section{Monachodesmus armorum sp.n.} Figs 1C, 6, 7.

HOLOTYPE $0^{7}$ (MRAC 22675), Cameroon, Ongot, $\mathrm{N}$ 0351', E $011^{\circ} 25^{\prime}$, open terrane, ca $810 \mathrm{~m}$ a.s.1., 19.VII.2014, leg. A. Nzoko.

PARATYPE: $10^{7}$ (MRAC 22674), Cameroon, Zamakoué, N $03^{\circ} 33^{\prime}$, E $011^{\circ} 31^{\prime}$, forest, ca $2320 \mathrm{~m}$ a.s.1., 19.IV.2014, leg. A. Nzoko.

NON-TYPE: 1 q (MRAC 22676), Cameroon, Campus Université Yaoundé 1, $\mathrm{N} 03^{\circ} 53^{\prime}$, E $011^{\circ} 30^{\prime}$, $860 \mathrm{~m}$ a.s.1., 28.IV.2014, leg. A. Nzoko.

NAME. To emphasize the unusual, shield-shaped, mesal plate of the gonopod telopodite (sh); noun in apposition.

DIAGNOSIS. Differs mostly by the relatively large size, the epiproct being fully exposed from above, as well as in the sheld-like mesal plate (sh) of the gonopod telopodite. See also Key below.

DESCRIPTION. Length ca 16-17, width of midbody pro- and metazonae $1.3-1.5$ and $2.4-2.5 \mathrm{~mm}$, respectively $\left(\sigma^{\top}, q\right)$. Holotype ca $17 \mathrm{~mm}$ long, 1.3 and $2.5 \mathrm{~mm}$ wide on pro- and metazonae, respectively. General coloration in alcohol light beige yellowish to light grey, usually with partly earth-encrusted, brown to grey-brown metaterga and dorsal crests (Fig. 1C). Vertigial paramedian regions above antennae usually yellowish brown.

Body with 20 segments $\left(\sigma^{\top},+\right)$. Body polydesmoid, with rather narrowly, poorly declivous, wing-shaped paraterga set very low (nearly level to venter) (Figs 1C, $6 \mathrm{~A}-\mathrm{J}$ ). Collum flabellate, very wide, fully covering the head from above; anterior margin pooly and regularly arcuate, with $5+5$ equal and rather faint lobulations; central region with $1+1$ high, basally rather clearly separated crests somewhat divided only subapically, the knob-shaped tips being single (only anteriorly, $\sigma^{7}$ ) or bifid (both anteriorly and posteriorly, + ); crests inclined more forward than dorsad, not overhanging collum's anterior margin; neither intercalary granulations nor (caudo)lateral lobulations (Figs 1C, 6A, H). Dorsum strongly convex, sides up to subvertical; dorsal crests (MD) on postcollum metaterga only slightly higher than lateral ones (DL), narrow, clearly separated, only on segments 3 and 4 bilobate, on other segments clearly trilobate; nearly not inclined caudad even on segments 17 and 18, much smaller on segment 19; intercalary granulations usually distinct, pattern typical of Pyrgodesmidae, i.e. these being arranged in two rows of $3+3$ between main crests and in 2-3 irregular rows of $3+3$ on sides below lateral crests; caudal intercalary granulations forming a row of small caudomarginals extending down to bases of paraterga, but leaving entire latter free from caudo- or anterolaterals (Figs $1 \mathrm{C}, 6 \mathrm{~A}-\mathrm{K})$. Pores or poroseles lying on caudal lobulation near caudal corner (Fig. 6K). Neither dorsal crests nor paraterga 19 considerably extended backwards, leaving epiproct fully exposed in dorsal view (Figs 1C, $6 \mathrm{C}, \mathrm{F}, \mathrm{J})$.

Legs short, barely reaching sides of paraterga, crassate, unmodified; gonapophyses vestigial (Fig. 6D-G, L).

Gonopods (Figs 6N, O, 7) rather simple; gonocoel rather deep, yet leaving much telopodite exposed; each telopodite deeply bipartite, lateral branch (I) long and slender, subacuminate, strongly exposed beyond gonocoel; mesal branch (m) prominent, consisting of a large, ribbed, rounded, shield-like structure (sh) on mesal face, a deeply bifid lobe (11 and 12) on caudal face at base of sh, and an abundantly fringed, slender, fingershaped solenomere (sl) lying between $\mathbf{l 1}$ and $\mathbf{s h}$.

REMARKS. The following key can serve to separate all 18 currently known Monachodesmus species. By necessity, the key is provisional, largely based on somatic chacters, because several species are only known from $q$ material, whereas the gonopod structure is often too complex to easily interpret.

1(2) Smaller intercalary granulations present at least between main crests, caudolaterals present. .................. 3

2(1) Smaller intercalary granulations on metaterga untraceable, caudolaterals absent. ......................................... 19 

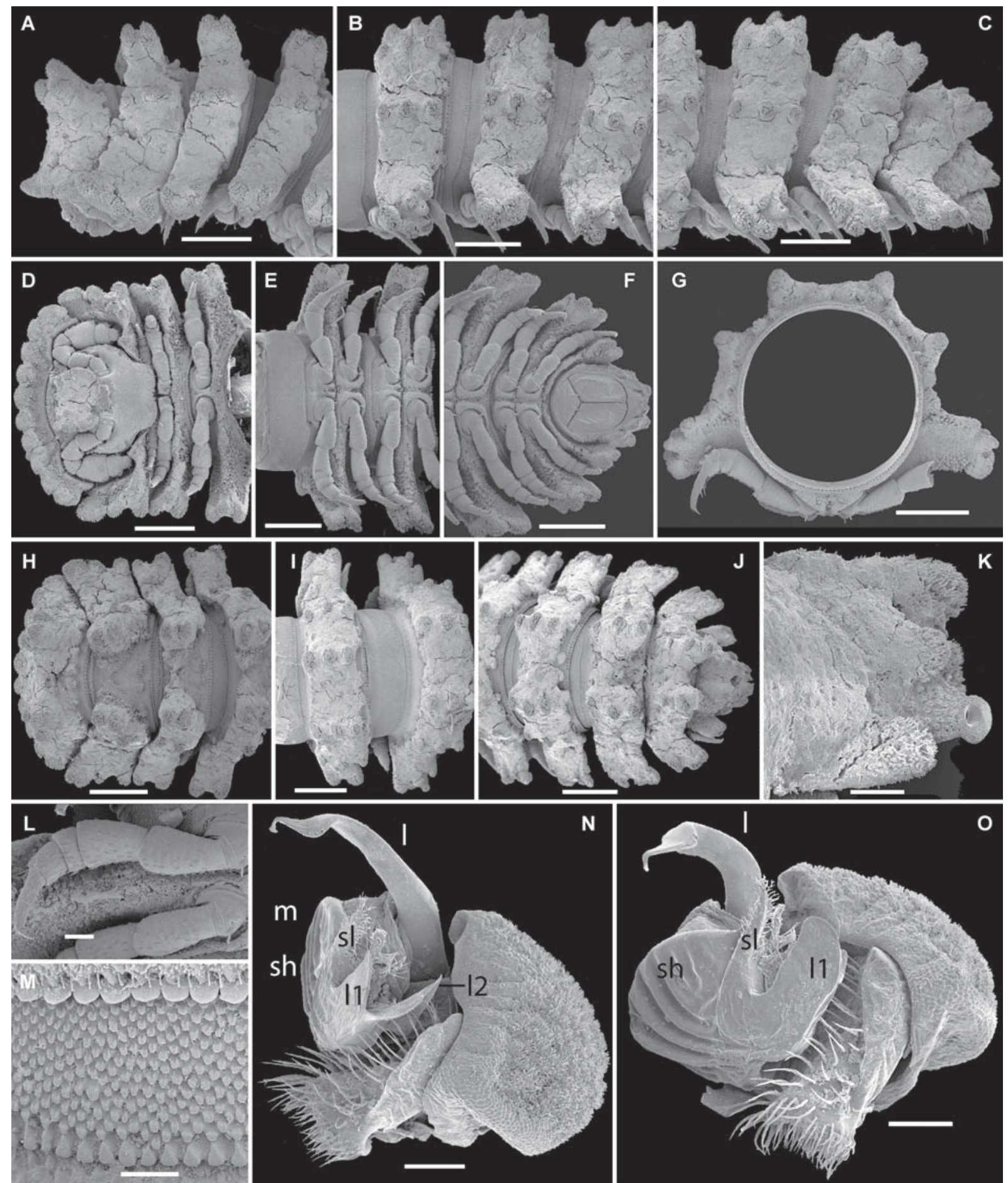

Fig. 6. SEM micrographs of Monachodesmus armorum sp.n., $\mathrm{O}^{7}$ paratype (A-M), $\mathrm{O}^{7}$ holotype (N \& O). A, D \& H - anterior part of body, lateral, ventral and dorsal views, respectively; B, E \& I - midbody segments, lateral, ventral and dorsal views, respectively; C, F \& $\mathrm{J}$ - posterior part of body, lateral, ventral and dorsal views, respectively; $\mathrm{G}$ - cross-section of a midbody segment, caudal view; $\mathrm{K}$ poriferrous paratergite with porostele, dorsal view; $\mathrm{L}$ — midbody leg, ventral view; $\mathrm{M}$ - limbus and adjacent microstructures; $\mathrm{N} \& \mathrm{O}$ left gonopod, caudal and mesal views, respectively (tip broken off). Scale bars: 0.5 (A-J), 0.1 (K. L, N, O) \& 0.05 (M). Designations in text.

Рис. 6. Фотографии SEM Monachodesmus armorum sp.n., паратип О (А-M), голотип О (N и O). A, D, Н — передняя часть тела, соответственно сбоку, снизу и сверху; В, Е, I - среднетуловищные сегменты, соответственно сбоку, снизу и сверху; C, F, J задняя часть тела, соответственно сбоку, снизу и сверху; $\mathrm{G}$ - поперечный срез среднетуловищного сегмента, сзади; К - несущий пору паратергит с поростелем, сверху; L — среднетуловищная нога; M - лимбус и смежные микроструктуры; N, O - левый гонопод, соответственно сзади и изнутри (вершина обломана). Масштаб: 0,5 (A-J), 0,1 (K, L, N, O) и 0,05 мм (M). Обозначения в тексте. 

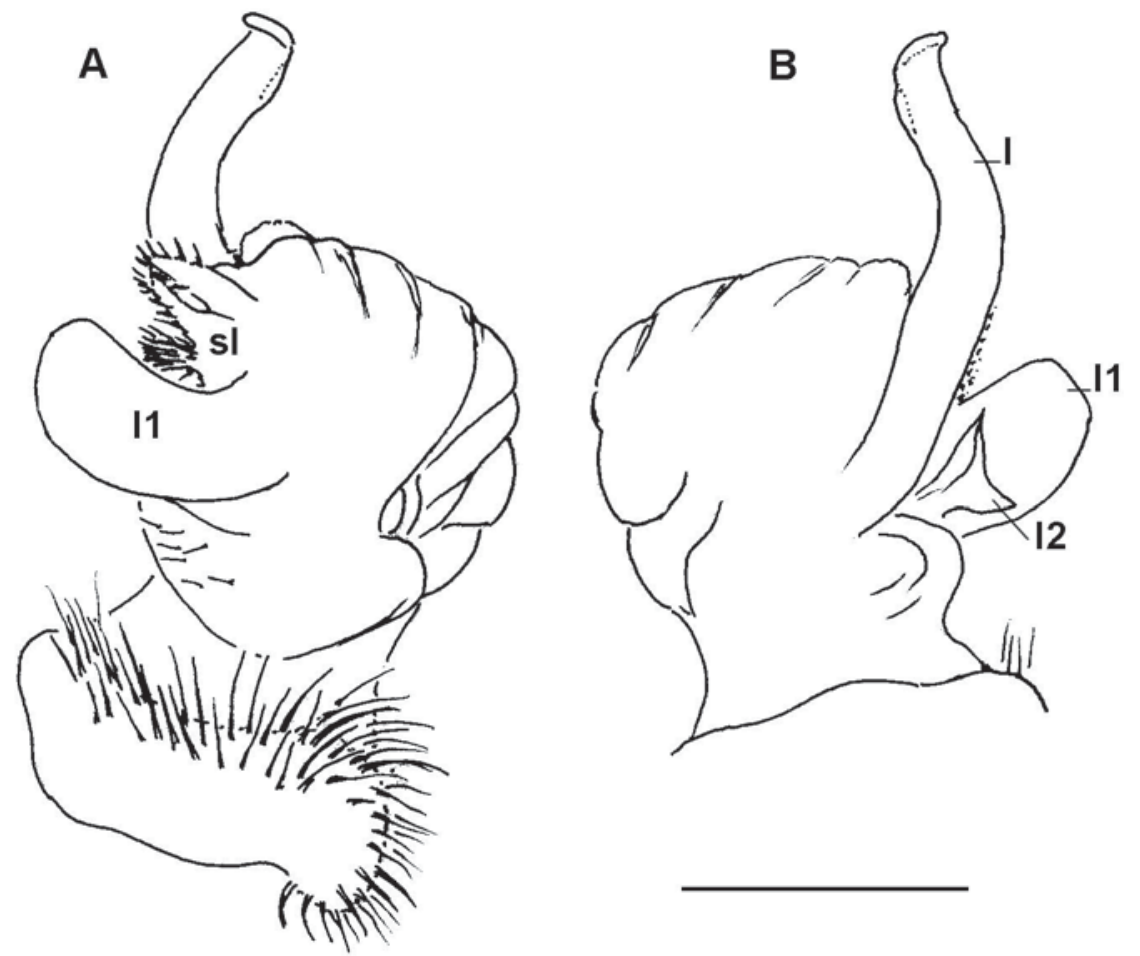

Fig. 7. Right gonopod of Monachodesmus armorum sp.n., O paratype. A \& B, mesal and lateral views, respectively. Scale bar: 0.2 $\mathrm{mm}$. Designations in text.

Рис. 7. Правый гонопод Monachodesmus armorum sp.n., паратип Оج. А и В, соответственно изнутри и сбоку. Масштаб: 0,2 мм. Обозначения в тексте.

3(4) Larger: adults $16-17 \mathrm{~mm}$ long. High, 1+1, at most slightly bifid, central tubercles on collum (Fig. 6A, H). Epiproct fully exposed in dorsal view (Figs 1C, 6C, F, J). Cameroon. M. armorum sp.n.

4(3) Smaller: length $<10 \mathrm{~mm}$. Several small, round tubercles in central part of collum, often including a few caudolaterals. Epiproct at least slightly, but fully concealing the epiproct in dorsal view.

5(6) Only postcollum metaterga with intercalary granulations, these being absent from collum

$6(5)$ Collum also with intercalary granulations, traceable at least as caudals and/or caudolaterals

7(8) Collum with $2+2$ and $2+2$ small, round tubercles arranged centrally in two transverse rows. Caudal tip of medially fused paraterga 19 deeply bilobed, notch nearly reaching the tip of epiproct. Cameroon. . M. spurcus

8(7) Collum with $2+2$ and $3+3$ small, round tubercles arranged centrally in two transverse rows. Caudal tip of medially fused paraterga 19 only rather faintly bilobed, notch far from reaching the tip of epiproct. Ghana. ..... M. conquisitus

9(10) Collum with $2+2$ and $2+2$ small, round tubercles arranged centrally in two transverse rows, plus $6+6$ smaller caudolaterals. Nigeria. M. angustus

10(9) Collum's ornamentation different. 11

11(12) Collum with two compact paramedian groups of $2+2$ and $2+2$ larger, rounded tubercles, plus a row of several smaller, grain-shaped caudals. Cameroon. .... M. lentus

12(11) Collum's ornamentation different. 13

13(14) Collum with $2+2$ and $3+3$ small, round tubercles arranged centrally in two transverse rows, plus at least several small caudals.
14(13) Collum's ornamentation different. 17

15(16) Ultimate dorsal crests less strongly elongated caudad, broadly and roundly bilobed, deeply split, not or barely concealing the epiproct from above. Guinea Bissau. .... M. subangustus

16(15) Ultimate dorsal crests more strongly elongated caudad, much narrower, lanceolate, subacuminate, very distinctly concealing the epiproct from above. Angola. M. albus

17(18) Collum with $3+3$ and $3+3$ small, round tubercles arranged centrally in two transverse rows, plus $6+6$ smaller caudals. Eritrea. M. escherichii

18(17) Collum with $2+2$ and $2+2$ small, round tubercles arranged centrally in two transverse rows, plus $2+2$ smaller caudolaterals. Zanzibar. .................. cristulifera

19(20) Porosteles extending caudally only until segment 15 , being absent from $16^{\text {th }}$. Angola. .............. lundanus

20(19) Porosteles present also on segment 16............... 21

21(22) Central tubercles on collum very high (Fig. 4B, E). Dorsal crests on segment 19 fused medially and extremely strongly elongate caudad, nearly as long as telson (Fig. 4D, G, J). Cameroon

M. longicaudatus sp.n.

22(21) Central tubercles on collum considerably lower. Dorsal crests on segment 19 never so strongly elongate caudad, usually at most half as long as telson. ......... 23

23(24) Collum with $2+2$ and $2+2$ small, round tubercles arranged centrally in two transverse rows. Epiproct only slightly concealed from above by ultumate dorsal crests.

25

24(23) Collum with $2+2$ and $3+3$ small, round tubercles arranged centrally in two transverse rows. Epiproct ei- 
ther exposed or concealed by ultumate dorsal crests in dorsal aspect. 27

25(26) Main dorsal and lateral crests poorly differentiated. Côte d'Ivoire and Guinea. ......................... M. odiosus

26(25) Main dorsal crests considerably higher than lateral ones. Cameroon. ................................. M. sexcarinatus

27(28) Main crests on postcollum metaterga inconspicuous, largely composed of small undifferentiated grains or knobs.

.. 29

28(27) Main crests conspicuous, clearly differentiated. . 31

29(30) Most of paraterga very narrow, subvertical, nearly wanting, only a few posterior ones broader and slightly explanate. Dorsal crests on segment 19 only slightly produced caudad, barely concealing the epiproct from above. Côte d'Ivoire.

M. unus

30(29) Paraterga broad and subhorizontal. Dorsal crests on segment 19 fused medially and very strongly produced caudad, about as long as telson. Principe Island, São Tome \& Principe. ............................................. M. feae

31(32) Dorsal crests on segment 18 strongly inclined caudad, in dorsal view almost reaching the ends of paraterga 19. Nigeria......................................................... M. tardus

32(31) Dorsal crests on segment 18 only slightly inclined caudad, in dorsal view at most reaching until midlength of paraterga 19.

33

33(34) Dorsal crests on segment 18 inclined caudad to reach until about midlength of paraterga 19, dorsal cones before tip of dorsal crests on segment 19 small, knobshaped. Gaboon. .......................................... M. silvestrii

34(33) Dorsal crests on segment 18 nearly not inclined caudad, dorsal cones before tip of dorsal crests on segment 19 larger, clearly elongate. Côte d'Ivoire. ....... 35

35(36) Ultimate cones before tip of dorsal crests on segment 19 drawn caudad clearly beyond notch dividing the tip. M. abijiensis

36(35) Ultimate cones before tip of dorsal crests on segment 19 reaching caudally only before notch dividing the tip. M. artatus

\section{Udodesmus camerunensis sp.n.}

Figs 1D, 8.

HOLOTYPE $0^{7}$ (MRAC 22677), Cameroon, Zamakoué, N $03^{\circ} 33^{\prime}$, E $011^{\circ} 31^{\prime}$, forest, ca $2320 \mathrm{~m}$ a.s.1., 28.VII.2014, leg. A. Nzoko.

NAME. To emphasize the terra typica; adjective.

DIAGNOSIS. Differs primarily in the undifferentiated lobulations at the anterior margin of the collum, as well as by the ozopore distribution pattern.

DESCRIPTION. Length ca $6 \mathrm{~mm}$, width of midbody pro- and metazonae 0.8 and $1.2 \mathrm{~mm}$, respectively $\left(\sigma^{\text {T }}\right)$. General coloration in alcohol grey-brown due to vertigial fields of granulations and, especially; collum and metaterga, most of head, including antennae, as well as prozonae, venter and legs nearly pallid (Fig. 1D).

Head smooth, roundish, genae square; clypeolabral region very finely and densely setose, epicranial suture visible only in occipital region; interantennal isthmus about twice as large as diameter of antennal socket; vertigial region with two elongated fields of fine microgranulations along epicranial suture. Antennae medium-sized, clavate, C-shaped, each lying inside a dis- tinct groove, closely appressed to head, with small compact apicodorsal groups of bacilliform sensilla on antennomeres 5 and 6 ; the former clearly the longest and largest.

Body with 20 segments $\left(\bigcirc^{7}\right)$. In width, head $<<$ collum $=$ segment $2<3<4<5=15$; thereafter body gradually tapering towards telson. Body polydesmoid, with rather broad, strongly declivous, wing-shaped paraterga set very low (nearly level to venter) (Figs 1D, E, 8). Collum flabellate, very wide, fully covering the head from above; anterior margin regularly arcuate, with $5+5$ equal and rather faint lobulations; central region with $2+2$ and $3+3$ distinct tubercles arranged in two transverse rows, these in anterior row being somewhat higher than those in posterior one; all tubercles clearly separated; neither intercalary granulations nor (caudo)lateral lobulations (Figs 1D, 8A). Lateral margin of paraterga 2 trilobate, following ones bilobate; sides strongly declivous to subvertical; dorsal crests (MD) on postcollum metaterga growing increasingly high towards $17^{\text {th }}$, then gradually, but clearly reduced towards $19^{\text {th }}$; MD bilobate on segments $2-4$, thereafter distinctly trilobate, only slightly inclined caudad in segments 17 and 18; lateral crests (DL) always trilobate, always shorter than MD, clearly reduced only in segments 18 and 19 (Figs 1D, 8A-D); starting with paraterga 2 , lateral margin mostly subrectangular at anterior and posterior corners; neither clear intercalary granulations nor caudolateral, nor anterolateral lobulations, nor tergal setae. Metaterga strongly convex, with a usual cerategument layer on top of microvilli, usually in part lightly encrusted also with earth material, with neither bosses nor areations. Pore formula normal: 5, 7, 9, 10, 12, 13, 15-19; very evident, coniform, simple porosteles present only in paraterga $5,7,9,10,12,13 \& 15$, in segments $16-19$ opening flush on tergal surface, always lying on caudal lobulation near caudal corner (Figs 1D, 8B-D). Limbus very finely crenulate. Epiproct nearly completely concealed from above, only tip seen between medially fused ultimate crests (Figs 1D, 8C, D). Hypoproct subtriangular, with $1+1$ caudal setae devoid of knobs.

Sterna narrow, deeply impressed along axis, without modifications, sparsely setose. Legs relatively short and stout, not extending beyond lateral edges of paraterga, only tarsi particularly slender; larger setae on $\sigma^{7}$ coxae modified, subplumose and ribbed (Fig. 1D). Gonapophyses on $\sigma^{7}$ coxae 2 vestigial.

Gonopod aperture subcordiform, broad, nearly as wide as prozona 7. Gonopods (Fig. 8E) rather simple; coxae subglobose, large, microgranulate laterally, with usual long cannulae and a rather deep gonocoel; telopodites in situ crossing medially, each deeply bipartite, basically consisting of a long, infuscate, curved, simple solenophore (sph) extending well beyond gonocoel and a somewhat shorter, also simple, rod-shaped solenomere (sl), the latter held perpendicular to $\mathbf{s p h}$ and lying on mesal face. 

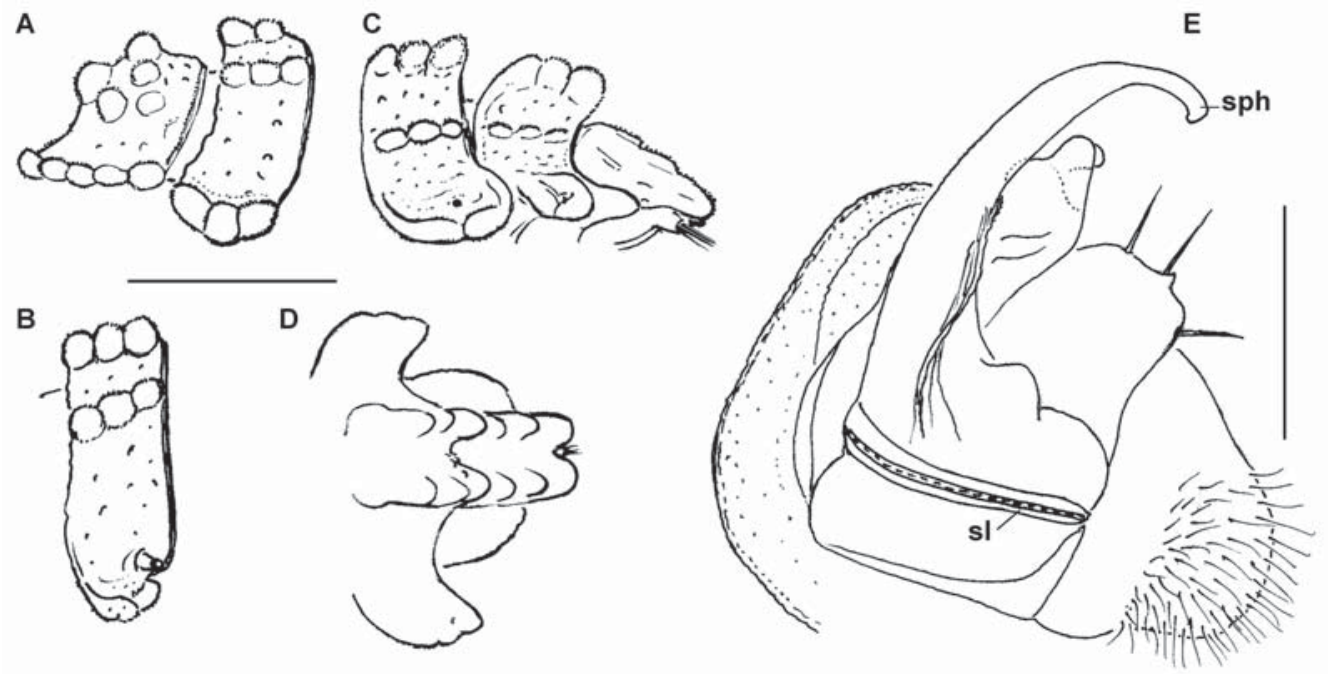

Fig. 8. Udodesmus camerunensis sp.n., $\mathrm{O}^{7}$ holotype. A - collum and segment 2, lateral view: B - segment 10, lateral view; C \& D caudal end of body, dorsal and lateral views, respectively; E - right gonopod, mesal view. Scale bars: 0.5 (A-D) \& 0.1 mm (E). Designations in text.

Рис. 8. Udodesmus camerunensis sp.n., голотип О7. А - коллум и сегмент 2, сбоку: В - сегмент 10, сбоку; С, D - задняя часть тела, соответственно сверху и сбоку; Е - правый гонопод, изнутри. Масштаб: 0,5 (A-D) и 0,1 мм (Е). Обозначения в тексте.

REMARKS. The West African genus Udodesmus Cook, 1896, has recently been reviewed and shown to comprise nine species, all keyed [Golovatch, VandenSpiegel, 2014]. One of the main diagnostic features of Udodesmus is the presence of clearly differentiated lobulations at the anterior margin of the collum. The new, and first, Udodesmus found in Cameroon is unique in showing these $5+5$ lobulations undifferentiated. However, the gonopod conformation which includes the long, strongly exposed, mesally crossing, rather simple solenophores often held perpendicular to similarly long and simple solenomeres leaves no doubt concerning the generic attribution.

ACKNOWLEDGEMENTS. The first two authors are greatly obliged to the Musée Royal de l'Afrique Centrale, Tervuren, Belgium for the invitation to join this project. Special thanks go to Jonathan Brecko (MRAC) for taking the colour pictures and to Christophe Allard (MRAC) for technical assistance.

\section{References}

Attems C. 1931. Myriopoden von Java, Sumatra und Bali // Archiv für Hydrobiologie. Suppl.-Bd.8. S.115-192.

Attems C. 1940. Myriopoda 3. Polydesmoidea III. Fam. Polydesmidae, Vanhoeffeniidae, Cryptodesmidae, Oniscodesmidae, Sphaeriotrichopidae, Peridontodesmidae, Rhachidesmidae, Macellolophidae, Pandirodesmidae // Das Tierreich. Lfg.70. S.i-xxxii+1-577.

Brolemann H.W. 1920. Myriapodes III, Diplopoda // Voyage de Ch. Alluaud et R. Jeannel en Afrique orientale (1911-1912). Résultats scientifiques. Paris: L. Lhomme. P.49-298.

Chamberlin R.V. 1951. On Diplopoda of North-East Angola // Museu do Dundo. Estudios Diversos (III). Lisboa. (Diamang Publicações Culturais No.10). P.65-93.
Cook O.F. 1896. Summary of new Liberian Polydesmoidea // Proceedings of the Academy of Natural Sciences of Philadelphia. Vol.1896. P.257-267.

Demange J.-M., Mauriès J.-P. 1975. Myriapodes-Diplopodes des monts Nimba et Tonkoui (Côte d'Ivoire, Guinée) récoltés par M. Lamotte et ses collaborateurs de 1942 à 1960. Etude systématique, caractérisation des diopsiulides africains, révision des Trachystreptini, essai de classification des Cordyloporidae // Annales du Musée Royal de l'Afrique Centrale, Tervuren. Série in $8^{\circ}$. Sciences Zoologiques. No.212. P.1-192.

Golovatch S.I., VandenSpiegel D. 2014. Notes on Afrotropical Pyrgodesmidae, 1 (Diplopoda: Polydesmida) // Arthropoda Selecta. Vol.23. No.4. P.319-335.

Hoffman R.L. 1999. Checklist of the millipeds of North and Middle America // Virginia Museum of Natural History Special Publication Number 8. Martinsville, VA. 584 pp.

Jorgensen M.C., Sierwald P. 2010. Review of the Caribbean pyrgodesmid genus Docodesmus Cook with notes on potentially related genera (Diplopoda, Polydesmida, Pyrgodesmidae) // International Journal of Myriapodology. Vol.3. P.25-50.

Kraus O. 1958. Diplopoden aus Angola // Museu do Dundo. Subsídios para o Estudo da Biologia na Lunda. Lisboa. Publicações Culturais da Companhia de Diamantes de Angola Diamang. No.38. P.47-84.

Mauriès J.-P. 1968 (for 1967). Matériaux récoltés par M. H. Coiffait au Gabon : Myriapoda, Diplopoda // Biologia Gabonica. T.3. Fasc.4. P.361-401.

Mauriès J.-P., Maurin M. 1981. Pyrgodesmidae et Cryptodesmidae (Diplopoda, Polydesmida) de la forêt de Téké, Côte d'Ivoire // Bulletin du Muséum national d'Histoire naturelle. Sér.4. T.3. Section A. No.1. P.187-202.

Porat O. 1894. Zur Myriopodenfauna Kameruns // Bihang till Kungliga Svenska Vetenskaps-Akademie. Bd.20. Afd.4. No.5. S. $1-90$.

Silvestri F. 1896. I diplopodi. Parte I - Sistematica // Annali del Museo Civico di Storia Natutrale di Genova. T.36. P.121-254.

Silvestri F. 1911. Descrizione di nouvi Polydesmoidae termitofili // Zoologischer Anzeiger. Bd.38. H.22/23. S.486-492.

Silvestri F. 1917. Contribuzione alla conoscanza dei termitidi e termitofili dell'Africa occidentale. II. - Termitofili (1) // Bollettino del Laboratorio di Zoologia generale e agraria della 
Reale Scuola superiore di Agricoltura di Portici. Vol.12. P.287346.

Silvestri F. 1927. Contribuzione alla conoscanza dei Polydesmidae (Diplopoda) dell'Africa occidentale // Bollettino del Laboratorio di Zoologia generale e agraria della Reale Scuola superiore di Agricoltura di Portici. Vol.20. P.287-323.

Verhoeff K.W. 1936. Ueber einige indische Chilognathen gesammelt von Herrn S. Jones, Madras // Records of the Indian Museum. Vol.38. Pt.2. P.103-123.
Verhoeff K.W. 1937. Zur Kenntnis indisch-ostasiatischer Diplopoden, Polydesmoidea // Zoologischer Anzeiger. Bd.120. H.11-12. S.306-319. 